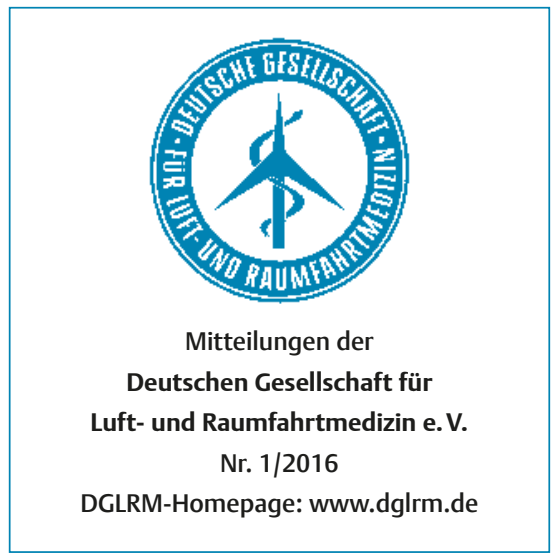

Geschäftsstelle der DGLRM

Christine Gens

Postfach 870204

13162 Berlin

E-Mail: christine.gens@dglrm.de

Tel.: 0176/5624-2876

\section{Wir gratulieren}

- 65 Jahre, Dr. Irene Hufnagel, Oestrich-Winkel, 22.02.1951

Wir begrüßen als neue Mitglieder

- Dr. Johannes Gudjohns, Gehrden

- Prof. Dr. Mike P. Holzer, Heidelberg

- Dr. Claus-Peter Limmer, Fürstenfeldbruck

- Dr. Dirk Müller, Langenhagen

- Dr. Lothar Zell, Ettlingen

\title{
Sehr geehrte Damen und Herren, liebe Mitglieder der DGLRM,
}

sehr wahrscheinlich wird das Jahr 2016 unserer Gesellschaft Entscheidungen abverlangen, die merkliche Veränderungen in der Flugmedizin in Deutschland und möglicherweise auch in der in Europa mit sich bringen könnten. Während unser „Pastpräsident“, Herr Professor Helmut Landgraf, vor ein paar Jahren in den DGLRM-Mitteilungen noch schreiben konnte, dass für Flugmediziner die Schatztruhe der Fortbildungsveranstaltungen und Kongresse reich gefüllt ist, mag dies für die Kongresse immer noch zutreffen, bei der Aus- und Fortbildung jedoch sehen wir uns gegenwärtig einer Situation gegenüber, die es angehenden Flugmedizinern sehr schwer machen wird, die von der EASA geforderten Ausbildungsinhalte in Deutschland vermittelt zu bekommen. Zum einen gibt es sehr viel weniger Ausbildungslehrgänge als in den Vorjahren und zum anderen sind diese bereits alle ausgebucht. Ich habe diese Situation bereits im letzten Heft der FTR angedeutet.

\section{Zukunft der Deutschen Akademie für Flugmedizin (DAF)}

Wie Sie ja bereits wissen, endet die Geschäftsführung von Herrn Professor Uwe Stüben, nach mehr als 25 Jahren sehr erfolgreicher Tätigkeit, mit der Vorlage des Jahresabschlusses für das Geschäftsjahr 2015 und seiner Entlastung voraussichtlich im Mai oder Juni dieses Jahres. Danach wird er dem neuen Geschäftsführer noch bis Ende 2016 zwecks Einarbeitung zur Verfügung stehen.

Da das ärztliche Personal des Medizinischen Dienstes der Lufthansa in Frankfurt, das bisher einen großen Teil der Ausbildung übernommen hat, aus den verschiedensten Gründen nicht mehr in der Lage ist, die angebotenen Lehrgänge auf ehrenamtlicher Basis weiterzuführen, muss nach Möglichkeiten gesucht werden, die flugmedizinische Ausbildung in Deutschland neu zu organisieren. Dazu gab es am 12. Januar eine außerordentliche Gesellschafterversammlung, um die aktuelle Situation zu erörtern und nach Lösungsmöglichkeiten zu suchen.

\section{Aktuelle Situation}

Die Lufthansa selbst, als bisherige Mehrheitsgesellschafterin (90\%), möchte in dieser Phase des Wechsels des Geschäftsführers als Hauptgesellschafterin ausscheiden und ihre Anteile weiter veräußern. Für eine Übergangszeit wäre für sie dabei durchaus eine kurzzeitige Beteiligung als Minderheitsgesellschafterin (10\%) vorstellbar.

Die Option einer Liquidation der Akademie und der Verteilung der Vermögenswerte im Sinne der Satzung an gemeinnützige Einrichtungen der Luftfahrtmedizin werden im Augenblick weder von Lufthansa noch von der DGLRM verfolgt.

Die favorisierte Lösung wäre der Erhalt der Akademie unter gleichzeitiger langfristiger Sicherung einer günstigen Perspektive und der bisher erreichten fachlichen Standards.

\section{Lösungsoptionen}

Zunächst haben sich 2 Organisationen (DLR und Universität Frankfurt) um die Weiterführung der Geschäfte der DAF beworben. Das DLR hat sich, nachdem bekannt wurde dass auch die Universität Frankfurt an einer Übernahme von Gesellschaftsanteilen interessiert ist, zurückgezogen. Bereits kurze Zeit später hat auch die Universität Frankfurt von einer Beteiligung abgesehen, da sie nicht in ihr Portfolio passen würde. Derzeit prüft das Universitätsklinikum Frankfurt, das wirtschaftlich unabhängig von der Frankfurter Universität ist, ob es sich in die DAF einbringen kann. Ein Konzept zur Fortführung der Akademie ist bereits vorgelegt worden. Weitere Interessensbekundungen sind auch von der Hessischen Landesärztekammer und der Universität Mainz eingegangen und werden gegenwärtig geprüft.

Gespräche mit dem Vorstand der European Society of Aerospace Medicine haben ergeben, dass auch die ESAM möglicherweise an einer Übernahme von Gesellschaftsanteilen interessiert sein würde. Zu den Fragen, ob dies tatsächlich möglich ist und wie sich dies praktisch verwirklichen lässt, wird der Vorstand der ESAM auf seiner nächsten Sitzung am 
ersten Wochenende im Februar eine Entscheidung herbeiführen.

Im Vorstand unserer Gesellschaft denken wir im Moment darüber nach, ob wir in einer solchen Konstellation unsere Gesellschafteranteile von bisher 10 auf $50 \%$ aufstocken sollten. Wir werden uns auf unserer Vorstandssitzung in der zweiten Februarhälfte dazu positionieren müssen. Eine tragfähige Entscheidung über die zukünftigen Gesellschafter der DAF soll auf der nächsten Gesellschaftersitzung im März 2016 erfolgen.

Ich halte Sie über die aktuellen Entwicklungen auf der Homepage auf dem Laufenden. An dieser Stelle nur noch so viel: Für das Jahr 2016 werden sowohl die Kurse „Basic“ und „Advanced“ als auch der Refresher-Kurs wie geplant stattfinden. Sie sind bereits ausgebucht. Die Termine für die Basic- und Advanced-Kurse finden Sie in diesem Heft und auf unserer Homepage sowie der der DAF. Für den Refresher-Kurs in Seeheim wird der Zeitpunkt baldmöglichst festgelegt und dann umgehend bekanntgegeben.

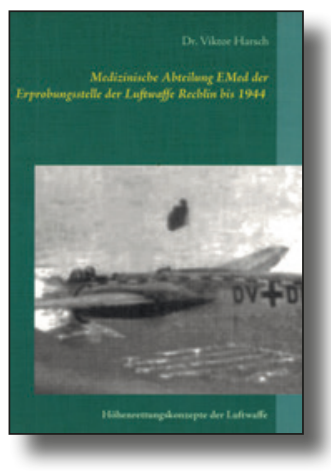

Neues Buch von

Dr. Viktor Harsch

Im Rethra Verlag Neubrandenburg ist als Fortsetzung der Flugmedizinischen Reihe der Arbeitsgruppe für die Geschichte der Luft- und Raumfahrtmedizin der DGLRM Ende letzten Jahres das neue Buch von Dr. Viktor Harsch zur Arbeit der medizinischen Abteilung der Erprobungsstelle der Luftwaffe (EMed) in Rechlin in den Jahren 1934-1944 erschienen.

Schwerpunkt der Abhandlung ist der Beitrag der EMed, deren Leiter der Flugmediziner Theodor Benzinger war, zur höhenphysiologischen Forschung bis Kriegsende und die Darstellung der Höhenrettungskonzepte der Luftwaffe. Der DGLRM wurde vom Autor ein Belegexemplar übergeben. Ich hatte bereits Gelegenheit, dieses Buch zu lesen und empfand die Lektüre über diese ereignisreiche Zeit nicht nur lehrreich, sondern kurzweilig, unterhaltsam und spannend zugleich. Ich kann sie Ihnen allen nur empfehlen und bedanke mich im Namen unserer Mitglieder bei Dr. Harsch ganz herzlich dafür!

\section{DGLRM-Jahreskongress 2016}

Hiermit möchte ich Sie sehr herzlich zu unserer diesjährigen Jahrestagung, der 54. in ihrer Folge, nach Bückeburg einladen. Sie findet vom 22. bis 24. September im dortigen Hubschraubermuseum statt. Das Programm wird dem multidisziplinären Charakter der Luft- und Raumfahrtmedizin Rechnung tragen und auch wieder

\section{Einladung und Call for Abstracts}

54. Jahrestagung der DGLRM

22.-24. September 2016

Hubschraubermuseum Bückeburg

Bitte reichen Sie Ihre Abstracts

für Vorträge und Posterpräsentationen

bis 31. März auf www.dglrm.de ein.

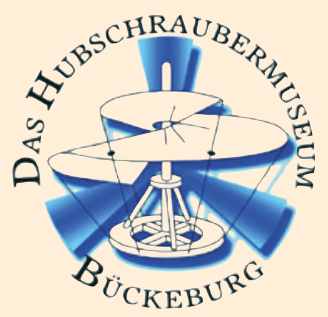

spannende Plenar- und Weiterbildungsvorträge sowie eine Fachbesichtigung und ein unterhaltsames wie auch lehrreiches Rahmenprogramm beinhalten.

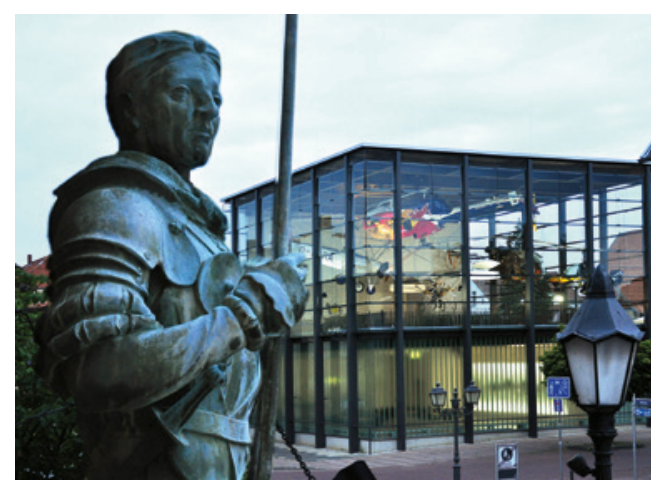

Bückeburg, Ort für die 54. Jahrestagung der DGLRM. Quelle: Hubschraubermuseum Bückeburg

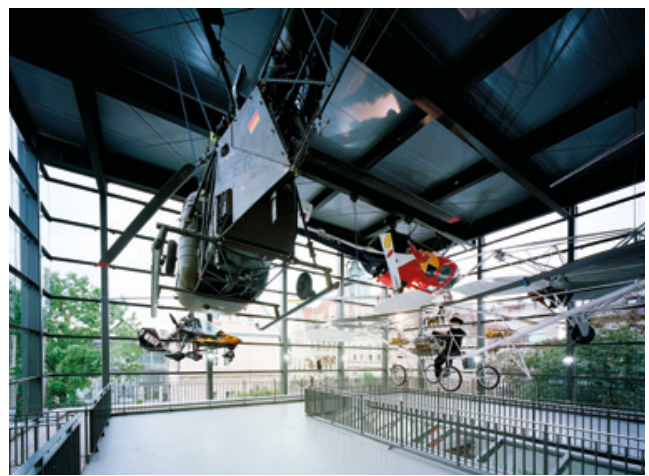

Blick auf ein Exponat des Museums.

Quelle: Hubschraubermuseum Bückeburg

Zur aktiven Mitwirkung bei der Programmgestaltung möchten wir Sie, wie jedes Jahr, zur Einreichung von Beiträgen auffordern und Ihnen damit Gelegenheit geben, der Fachwelt Ihre neu gewonnenen Ergebnisse und Erkenntnisse in Vorträgen und Postern zu präsentieren und zur Diskussion zu stellen. Wir freuen uns auf Ihre aktive Teilnahme, lebhafte wissenschaftliche Diskussionen, einen angeregten persönlichen Austausch sowie spannende Tage in der reizvollen Stadt Bückeburg!

Bitte reichen Sie Ihre Abstracts für Vorträge und Posterpräsentationen bis zum 31. März 2016 ein! Das Abstractformular finden Sie auf der Homepage. Wie es bei uns bereits langjährige Tradition ist, werden die besten Poster wieder mit Preisen gewürdigt. Weitere Details entnehmen Sie bitte unserer Homepage www.dglrm. de. 


\section{Kongresse 2016}

ÖGLM-Jahrestagung 2016

Österreichische Gesellschaft für Luftfahrtmedizin

Termin: 16.04.-17.04.2016

Ort: Alpenflugschule Niederöblarn, Österreich

Internet: www.flugmedizin.net

$87^{\text {th }}$ AsMA Annual Scientific Meeting

Termin: 24.04.-28.04.2016

Ort: Atlantic City, USA

Internet: www.asma.org
61. Arbeitstagung der Fliegerärzte der Bundeswehr Termin: 06.06.-09.06.2016 Ort: $\quad$ Fürstenfeldbruck Nur für aktive Fliegerärzte der Bundeswehr offen

ECAM 5 - $5^{\text {th }}$ European Conference of Aerospace Medicine

Scientific Conference of the European Society of Aerospace Medicine Termin: 15.09.-18.09.2016 Ort: Oslo, Norwegen Internet: www.esam.aero
54. Jahrestagung der Deutschen Gesellschaft für Luft- und Raumfahrtmedizin

Termin: 22.09.-24.09.2016

Ort: Hubschraubermuseum Bückeburg

Internet: www.dglrm.de

$64^{\text {th }}$ International Congress of Aviation and Space Medicine (ICASM) International Academy of Aviation and Space Medicine

Termin: 06.11.-10.11.2016

Ort: $\quad$ New Delhi, Indien

Internet: www.icasm.org

\section{Verantwortlich für den Inhalt}

\section{der DGLRM-Seiten}

Deutsche Gesellschaft für Luft- und Raumfahrtmedizin e. V. (DGLRM)

OFA PD Dr. Carla Ledderhos, Präsidentin Zentrum für Luft- und Raumfahrtmedizin der Luftwaffe

Str. der Luftwaffe 322

82242 Fürstenfeldbruck

carlaledderhos@bundeswehr.org

Bankverbindung

Deutsche Bank Hamburg

Konto-Nr.: 388948200

BLZ: 20070000

IBAN: DE 63200700240388948200

BIC: DEUTDEDBHAM

Änderungen für die Mitgliederkartei bitte an: Dipl. Ing. Christine Gammel

Zentrum für Luft- und Raumfahrtmedizin

der Luftwaffe

Str. der Luftwaffe 322

82242 Fürstenfeldbruck

christinegammel@bundeswehr.org 